\title{
MRI-guided stereotactic amygdalohippocampectomy: a single center experience
}

\author{
This article was published in the following Dove Press journal: \\ Neuropsychiatric Disease and Treatment \\ II February 2015 \\ Number of times this article has been viewed
}

\section{Zdeněk Vojtěch' \\ Hana Malíková ${ }^{2}$ \\ Lenka Krámská ${ }^{3}$ \\ Roman Liščák ${ }^{4}$ \\ Vilibald Vladyka ${ }^{4}$}

'Department of Neurology, ${ }^{2}$ Department of Radiodiagnostics, ${ }^{3}$ Department of Psychology, ${ }^{4}$ Department of Stereotactic and Radiation Neurosurgery, Na Homolce Hospital, Prague, Czech Republic
Correspondence: Zdeněk Vojtěch Department of Neurology, Na Homolce Hospital, Roentgenova 2, I50 30 Prague 5, Czech Republic

Tel +420 257272556

Fax +4202572733I4

Email zdenek.vojtech@homolka.cz
Background: This paper presents our experience of stereotactic radiofrequency amygdalohippocampectomy performed for intractable mesial temporal lobe epilepsy.

Methods: The article describes the cases of 61 patients who were treated during the period from 2004 to 2010. Mean postoperative follow-up was 5.3 years. Neuropsychological results were obtained for a subset of 31 patients.

Results: At their last postsurgical visit, 43 (70.5\%) patients were Engel class I, six (9.8\%) class II, nine (14.8\%) class III, and three (4.9\%) class IV. The surgery was complicated by four intracranial hematomas. One of them caused acute hydrocephalus and was treated by shunting and resolved without sequelae, while another caused transitory aphasia. The remaining hematomas were asymptomatic. There were two cases of meningitis which required antibiotic treatment. One patient committed suicide due to postoperative depression. After the procedure, we performed open epilepsy surgery and rethermolesions in three and two patients, respectively $(8.2 \%)$. Patients showed increases in their mean full scale, verbal, and performance intelligence quotient (IQ) scores of 4, 3, and 4 IQ points, respectively $(P<0.05)$. Five (17.2\%), four (13.8\%), and four (13.3\%) patients improved their full scale, verbal, and performance IQ scores, respectively. No significant changes were found in memory performance, with a mean increase of 1,3 , and 0 memory quotient points in global, verbal, and visual memory, respectively $(P<0.05)$. Global memory improved in three $(10.3 \%)$ patients, verbal memory in one $(3.4 \%)$, and one patient (3.3\%) showed deterioration in visual memory.

Conclusion: Stereotactic radiofrequency amygdalohippocampectomy offers a safe, effective, and less aggressive treatment modality in cases of well-defined mesial temporal lobe epilepsy. Seizure outcome is comparable with the results of conventional epilepsy surgery and cognitive results could be even better.

Keywords: temporal lobe epilepsy, epilepsy surgery, neuropsychology, hippocampal sclerosis, seizure outcome

\section{Introduction}

Mesial temporal lobe epilepsy (MTLE) is the most common pharmacoresistant epilepsy in adults, and hippocampal sclerosis (HS) is the most common substrate responsible for epileptogenesis among these patients. ${ }^{1}$ A relatively restricted epileptogenic zone and frequent intractability makes MTLE/HS the most surgically amendable epileptic syndrome. ${ }^{2}$ Surgical management has evolved from temporal lobe resection to more restricted procedures. These include Spencer type anteromesial temporal lobectomy (AMTR) with more extensive mesial resection and limited ablation of the temporal neocortex ${ }^{3}$ and several variants of amygdalohippocampectomy (AHE). ${ }^{4,5}$ These procedures have been shown to produce long-term cure rates of approximately $60 \%-80 \%{ }^{6}$ Most authors have found that patients with complete resections of the mesial temporal structures (hippocampus, parahippocampal gyrus [PHG], and, possibly, the amygdala) have the best chance of 
seizure-free outcomes. ${ }^{7}$ However, physicians may be reluctant to consider surgery for MTLE due to concerns over undesirable side effects and surgical complications.

As potentially functional brain tissue is resected, neuropsychological deficits in patients with MTLE are frequently aggravated or develop de novo following surgery. Mesial temporal structures (the hippocampus and the PHG) are important for the encoding and retrieval of information. Memory deterioration is a known adverse effect of the resection of these structures. The most feared complication is verbal memory decline after operations on the language-dominant side, which is reported in $22 \%-63 \%$ of these patients. ${ }^{8}$ It has been hypothesized that some of cognitive changes may be caused by collateral trauma, persistent seizures, and mood disorders. Functional release due to seizure freedom counteracts this negative impact in patients capable of developing compensatory strategies. ${ }^{9}$ Some studies have reported improved neuropsychological outcomes after more restricted operations. ${ }^{10}$

There may be speech worsening (impaired word finding and naming) after dominant side operations, which may persist in one out of four operated patients 1 year after surgery. This may be caused by vasospasms or brain contusion. ${ }^{11}$

Psychiatric disorders (depression, psychosis) may be present in one half of patients before the operation. Postoperatively, these disorders may worsen or appear de novo, especially during a period of several months following the operation..$^{12}$

A common complication of temporal resections involves upper quadrant visual field defects ("pie in the sky") due to damage to the Meyer's loop. Although their prevalence is high, patients usually do not notice them during formal visual field evaluations. Complete homonymous hemianopia is rare. ${ }^{13}$

Surgical complications are relatively infrequent in temporal lobe epilepsy surgery. ${ }^{14}$ Minor complications occur in $3.6 \%-15.2 \%$ of cases, and permanent morbidity is $1 \%-2 \%$ in most centers. A typical transitory complication is hemiparesis, which is thought to result from ischemia, brain edema, contusion, or hemorrhage. Meningitis occurs in $1.4 \%$ and cutaneous flap infections in $3.5 \%$ of patients. The main cause of persistent hemiparesis is ischemic capsular stroke, and this was found in $0.84 \%$ of patients. In most cases it results from manipulation of the anterior chorioidal artery, with subsequent infarction of the posterior limb of the internal capsule.

In some patients general medical conditions (deep venous thrombosis, pulmonary embolism) may occur, probably as a consequence of the surgical burden and hospital stay.
The search for surgical techniques which may obviate these complications is therefore justifiable. ${ }^{15}$ Ideally, these techniques should 1) eliminate unnecessary cortical and white matter tract trauma, 2) avoid manipulation of the cerebral vessels and cranial nerves, 3) attack as little of the mesial temporal structures as is necessary for seizure relief while leaving a maximum of potentially functional tissue undisturbed, and 4) be a relatively simple and safe procedure with a short hospital stay.

Stereotactic epilepsy surgery has a long history. Originally, these procedures (fornicotomy and anterior commissurotomy) focused on the improvement of behavioral disorders in epileptic patients and the prevention of seizure spread. ${ }^{16}$ It was subsequently noted that stereotactic amygdalotomy reduced seizure frequency in certain patients. A $50 \%$ reduction of seizure frequency was reported after stereotactic lesions in the amygdala and hippocampus, but the seizure-free rate was low (10\%). ${ }^{17}$ Amygdalohippocampal stereotactic lesions using a trajectory passing through the long axis of hippocampus were first created by brachytherapy. ${ }^{18}$ This treatment modality was limited by the risk of radiation injury to the adjacent blood vessels. During the period from 1967 to 1976, Vladyka used a string electrode construction resembling our device. ${ }^{19}$ He performed stereotactic coagulations of the amygdala and hippocampus in 38 patients. Target structures were localized by ventriculography and stereotactic atlas. He introduced the electrode along the long axis of hippocampus and, by heating the electrode tip to $70^{\circ} \mathrm{C}$, performed 18-22 segmental coagulations. In this way he created an elongated lesion $25-30 \mathrm{~mm}$ long and $10 \mathrm{~mm}$ in diameter. Of 22 patients with a follow-up of $\geq 2$ years he reported seizure-free outcomes in $50 \%$. Thirty-two percent experienced significant improvement, and in $18 \%$, seizure frequency was unchanged. Over the same time period and in the same institution the results of open surgery were $52.3 \%$ seizure-free, $19.1 \%$ significantly improved, and $28.6 \%$ unchanged. Thus, epileptological results were comparable with contemporary open surgery. Complications were as follows: one intracerebral bleeding, two abscesses, one hemiparesis, and one homonymous hemianopia. In case of treatment failure, patients were eligible for subsequent operation.

The introduction of modern neuroimaging into the evaluation of epilepsy encouraged renewed interest in stereotactic surgery for the treatment of MTLE. Initial experiences with small series of patients were encouraging. Kratimenos et al described the disappearance of seizures or the marked reduction of their frequency with minimal 
morbidity in a group of six patients using open stereotactic AHE. ${ }^{20}$ Patil et al reported their experience with nine patients in whom computerized tomography-navigated stereotactic radiofrequency amygdalohippocampectomy (SAH) (seven patients) and corpus callosotomy (two patients) were combined with multiple subpial transections (six patients). ${ }^{21}$ Five patients (56\%) were seizure-free, three $(33 \%)$ had greater than $90 \%$, and one $(11 \%)$ greater than $50 \%$ reduction in seizure frequency during a median 19-month follow-up. There were no complications except for temporary hemiparesis attributable to multiple subpial transections in one patient.

SAH was reintroduced by a London, Ontario group as an alternative therapy for MTLE. ${ }^{22}$ A coagulation electrode was introduced using a lateral approach through burr holes placed over a medial temporal gyrus. They defined two lesion groups in whom they created discrete (group I: five patients) and confluent (group II: 15 patients) lesions. ${ }^{23}$ One patient $(20 \%)$ in group I and nine (60\%) in group II experienced a favorable seizure outcome. Two patients (both in group II) were seizure-free. Magnetic resonance imaging (MRI) scans at 6-9-months follow-up disclosed discrete areas of atrophy in the amygdala and hippocampus, interspersed with preserved brain in the group I patients. More uniform and complete destruction of the amygdala and hippocampus was evident in group II patients. All lesions were confined to the amygdala and hippocampus, sparing the PHG. The authors concluded that the extensive amygdalohippocampal ablation in group II improved seizure outcome, but these results were inferior to open surgical approaches; only $10 \%$ of treated patients were seizure-free. They speculated that the poor results were caused by epileptogenicity of the PHG, which was not destroyed by their technique.

This article is an overview of our experience with SAH, which was acquired over time. We summarize our SAH technique, outcomes with respect to seizures, neuropsychological results, and complications. We try to compare our results with outcomes from conventional epilepsy surgery. Finally, we speculate on the possible advantages of the incomplete destruction of the target structures.

\section{Materials and methods Participants}

Over the period from 2004 to 2010, 61 patients (31 male and 30 female) were treated for MTLE by SAH at our institution. ${ }^{24}$ The group was rather heterogenous. Mean start of epilepsy was 16.6 years (median 13, range $1-54$ years), mean duration of epilepsy until surgery was 24.5 years (median 23, range 2-73), and mean age at the time of surgery was 41.3 years (median 38, range 17-75).

In all but two patients SAH was the first resective surgery. In one patient, SAH without a modern diagnostic work-up had been performed in the 1970s, and the target structures were partially missed. In one patient, stereotactic lesion was targeted to hippocampal remnants found after AMTR performed at another institution. In two patients, implantation of a vagus nerve stimulator was performed 1 and 3 years before SAH, respectively. The device was explanted 3 and 6 years thereafter, respectively. In one patient, periventricular cavernoma was treated by Leksell gamma knife radiosurgery 3 years before SAH, with no change in seizure frequency. Further information on this section can be found in Table S1 and in previous work. ${ }^{24}$

\section{Preoperative testing}

Each patient underwent a routine presurgical examination (evaluation by at least one certified epileptologist, neuropsychological examination, Wada test, long-term videoelectroencephalogram monitoring with scalp electrodes placed according to the international 10-20 system, and MRI using a dedicated epilepsy protocol). All but three patients underwent positron emission tomography (PET) scans. An invasive video-electroencephalogram study was performed on two patients.

MRI scanning was performed using a 1-Tesla whole-body MRI system. ${ }^{25}$ In addition to common sequences (turbo spin echo [TSE] T2-/proton-density-weighted imaging axial, T2-weighted imaging [WI] turbo fluid-attenuated inversion recovery, axial and coronal, TSE T2-WI coronal and spin echo, T1-WI sagittal plane; coronal planes were orientated perpendicularly to the long axis of the hippocampus), a three-dimensional volume acquisition sequence (FLASH 3 dimensions, repetition time $25 \mathrm{~ms}$, echo time $5 \mathrm{~ms}$, flip angle $37^{\circ}$, slice $1.3 \mathrm{~mm}$, matrix size $256 \times 256$ ) was obtained. In selected patients, MRI volumetry of the hippocampus and of the amygdala was performed by an experienced radiologist who was not provided with any clinical information. Volumes were measured from three-dimensional scans obtained preoperatively and 1 year after treatment. Anatomical landmarks were determined by a well-defined protocol. ${ }^{26,27}$

Patients underwent neuropsychological tests in two sessions over two consecutive days, each lasting 60-90 minutes. ${ }^{28}$ During the first day, a psychological interview was performed, and the Wechsler Adult Intelligence Scale-Revised test was presented. Memory, verbal functions, and quality of life were assessed on the second day using the Wechsler Memory 
Scale-Revised test, a verbal fluency test (standardized Czech versions), and a Quality of Life in Epilepsy Inventory (QOLIE-89), respectively.

Full convergence of noninvasive data (MRI, PET, and scalp video-electroencephalogram) was found in 36 patients. Twenty-two patients had incomplete convergence or incomplete data (absent PET). Besides HS, additional potentially epileptogenic MRI-lesions were found in three patients (cortical dysplasia in the PHG ipsilateral to HS, bilaterally dysplastic occipital cortex, and subependymal cortical periventricular heterotopia in the frontal region ipsilateral to HS in each case). In one patient, marked diffuse brain atrophy was noted. Unrelated lesions were found in two patients (pituitary adenoma and fusiform aneurysm of the basilar artery). Based on these results, we indicated $\mathrm{SAH}$ on the left in 41 and on the right in 20 patients. Further information on this section can be found in Table S2 and in a previous report. ${ }^{24}$

After completion of the preoperative evaluation, therapeutic options (open surgery and $\mathrm{SAH}$ ) were suggested to the patients, who then chose one of them. All subjects were fully informed and gave their informed consent. The SAH technique was approved by the local ethics committee.

\section{Technique for the stereotactic procedure}

SAH was carried out under local anesthesia and mild sedation, with the patient in a semiseated position..$^{29}$ Before starting the procedure, an intravenous bolus of antibiotics was administered to all but the first two patients, and a circle of hair was shaved occipitally. The Leksell stereotactic system (Elekta Instruments, Sweden) was used, and the coordinate frame was attached to the patient's head. MRI was performed with an indicator box using the $1.0 \mathrm{~T}$ (Siemens Impact) or $1.5 \mathrm{~T}$ (Siemens Avanto) MRI system, with TSE T2-WI coronal, slice $2 \mathrm{~mm}$ and three-dimensional volume acquisition sequence (FLASH 3D, TR $25 \mathrm{~ms}$, TE $5 \mathrm{~ms}$, flip angle $37^{\circ}$, slice $1.3 \mathrm{~mm}$, matrix size $256 \times 256$ ) with the administration of an intravenous gadolinium contrast agent to visualize the cortical vessels. Thermocoagulation of the amygdalohippocampal complex with the entry point in the occipital region and a single trajectory was planned according to the individual anatomy of the patient using SurgiPlan (Elekta Instruments) (Figure 1). Maximum efforts were made to avoid the ependymal surface of the ventricle and the cortical vessels. A percutaneous drill hole was made under local anesthesia (diameter of drill $4 \mathrm{~mm}$ ), and the dura mater was penetrated with the coagulating tip of the probe. SAH was

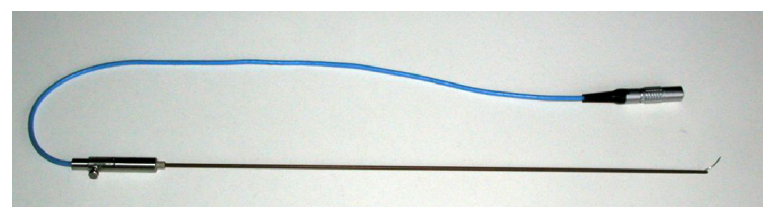

Figure I String electrode with exserted $10 \mathrm{~mm}$ bold active tip.

carried out using a device with a $1.8 \mathrm{~mm}$ outer diameter of the guiding tube and the flexible $10 \mathrm{~mm}$ bold active tip (Neuro N50; Fischer-Leibinger, Germany) (Figure 2) was inserted and guided through a trajectory passing the hippocampal head to the coordinates of the target located in the amygdala. An individual lesion was made when the string tip of the device was exserted from the guiding tube to its side, reaching $8 \mathrm{~mm}$ lateral to the long axis of the probe. The active tip was heated for 60 seconds to $75^{\circ} \mathrm{C}$ (in 15 patients) or $88^{\circ} \mathrm{C}$ (in the remaining 46 patients) depending on the probe's thickness (two versions of electrodes with different thicknesses of the string tip were used). When thermocoagulation was accomplished, the active tip was retracted, the electrode was rotated by $45^{\circ}$ according to the treatment plan, and the tip was again exserted to create another lesion. Usually, two to four lesions were made in each position. After lesioning had been completed in one position, the tip was retracted and the probe withdrawn by $5 \mathrm{~mm}$ to the level of the next position. Thermocoagulation was performed in seven to ten positions, making a confluent lesion $30-45 \mathrm{~mm}$ in length. The number of lesions in all positions ranged from 16 to 38 . The patients spent about 1 hour in the operating theater; the whole procedure from frame attachment to its removal from the patient's head usually lasted about 3 hours.

\section{Follow-up}

After the procedure, patients were hospitalized in a standard neurological ward, and early complications were recorded during this period. MRI verification of the size of the lesions was carried out before they were discharged. Neuropsychological reevaluation was performed at least 1 year after surgery. Seizure outcome was assessed at yearly intervals using Engel's classification. Mean postoperative follow-up was 5.3 years (median 5 , range $1-8$ ).

Seizure outcome was recorded for all patients. For seizure assessment, we used Engel's outcome classification for 2 years after surgery prior to each follow-up visit. As some patients were referred for the treatment from other institutions, neuropsychological outcomes were only available in subgroups of patients. Volumetry was performed in patients 
A
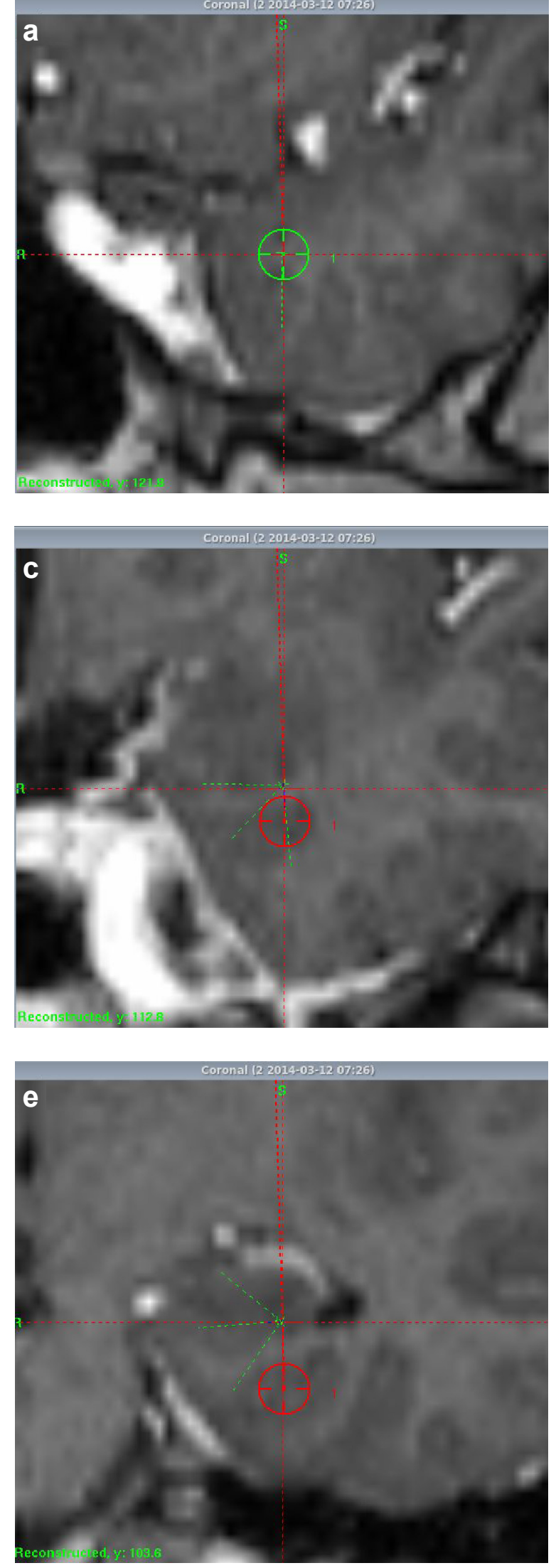
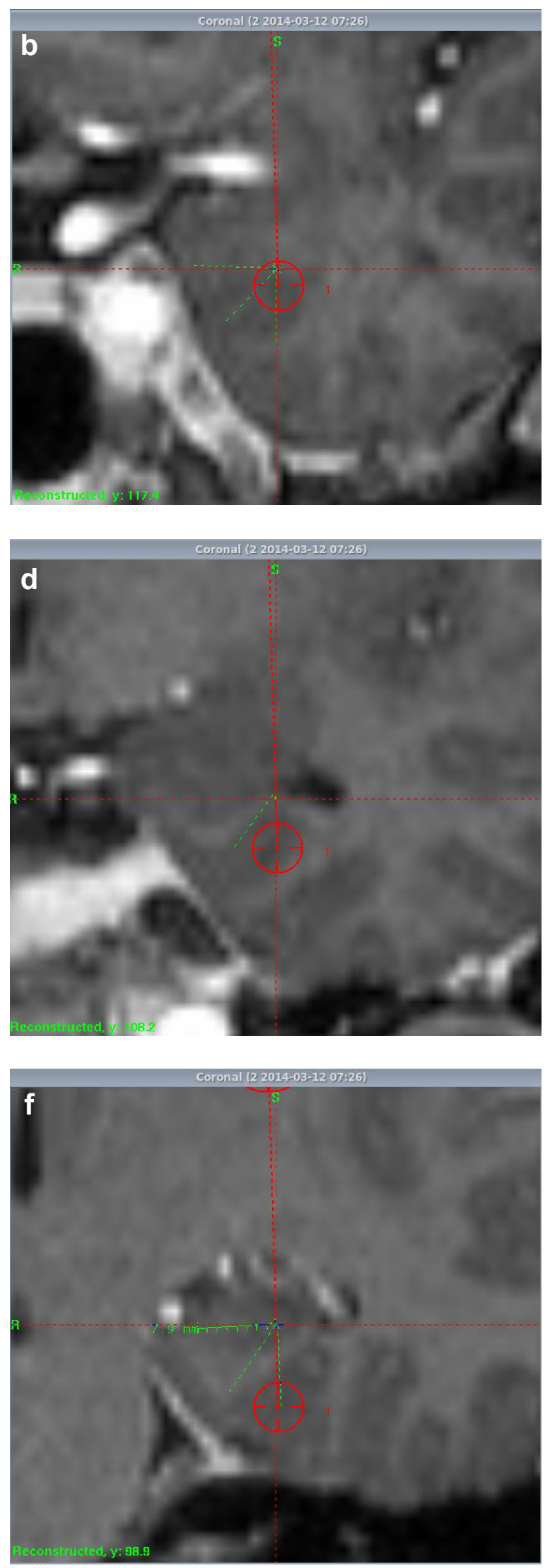

Figure 2 (Continued) 

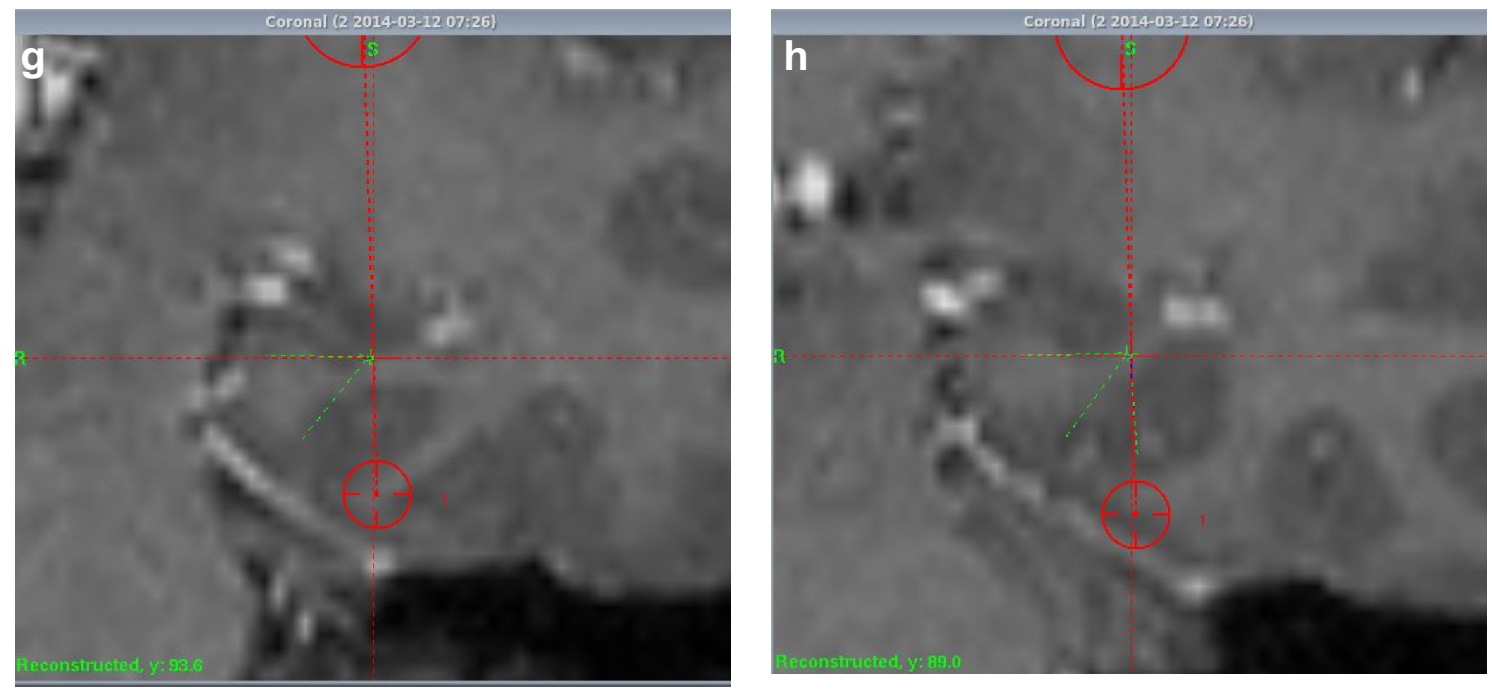

B

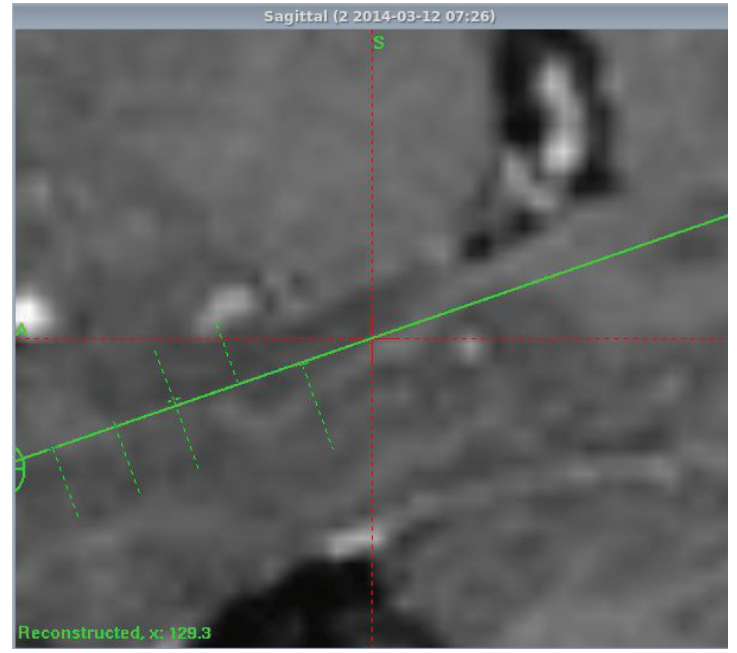

C

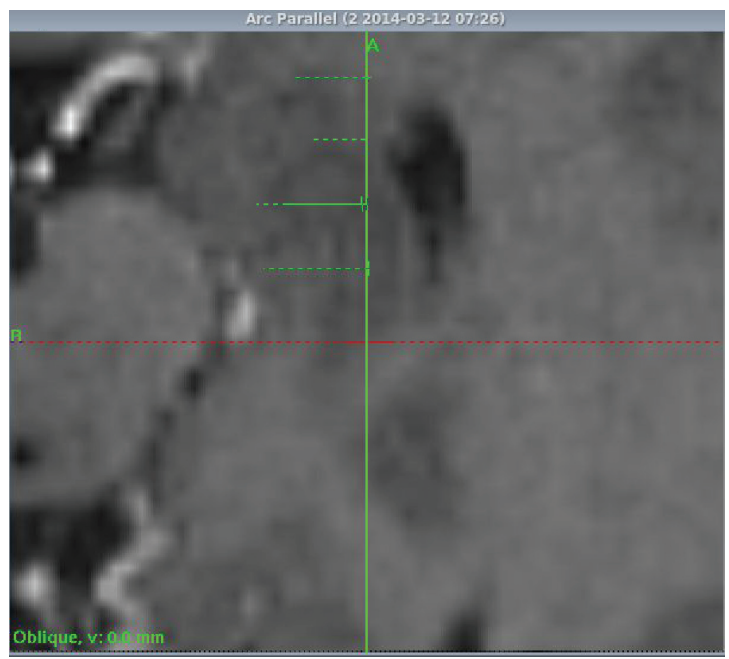

Figure 2 Treatment plan.

Notes: The pictures series in (A) show the planning of the probe tip extrusion in eight positions at various levels of the amygdalohippocampal complex in the coronal plane $(\mathrm{a}-\mathrm{h})$, and pictures $(\mathbf{B})$ and $(\mathbf{C})$ show the trajectory from a lateral and axial view.

with a sufficient follow-up period at the time of the study, and volumetric data of various mesial temporal structures were also calculated for smaller subsets of patients.

\section{Results}

\section{Morphologic outcome}

The early postoperative MRI scans showed coagulation lesions with heterogeneous signal intensity on both T1- and T2-WI, encompassing a significant part of the amygdala, hippocampus, and PHG. ${ }^{29}$ They contained hemorrhagic foci and were surrounded by vasogenic edema. After gadolinium contrast administration, peripheral ring enhancement occurred. The size of the lesions was $48.3 \pm 5.6 \mathrm{~mm}$ in their long axis and $20.5 \pm 3.6 \mathrm{~mm}$ in their short axis. MRI 1 year after the procedure showed postnecrotic oblong pseudocysts surrounded by atrophic tissue with gliosis. We did not find any long-lasting edema or contrast enhancement on follow-up. The mean size of pseudocysts was $32.2 \mathrm{~mm}$ in their long axis and $9.5 \mathrm{~mm}$ in their short axis (Figure 3).

\section{Outcome with respect to seizures}

At the last postsurgical follow-up, 43 (70.5\%) patients were Engel class I, six (9.8\%) class II, nine (14.8\%) class III, and three (4.9\%) class IV. ${ }^{24}$ Results are summarized in Table 1 . Two patients had not completed their 2-year follow-up visit; one died after an accident, and in the other, AMTR was performed.

Statistical analysis showed no difference in epilepsy duration $(P=0.1083)$ and age at the time of operation $(P=0.718)$ between classes II-IV and class I patients. Neither did we find any statistical difference between patients with completely and incompletely congruent preoperative results $(P=0.5215)$. 
After SAH, we performed AMTR in three patients. They were Engel class IIA, IVB, and IIIA for 2, 1, and 3 years after $\mathrm{SAH}$, respectively. They subsequently became Engel IA for 5,6 , and 1.5 years, respectively. Histopathological examination of the resected tissue revealed foamy macrophages infiltrating the walls of pseudocysts and neoformation of blood vessels. Surviving neurons were present within the pyramidal layer of the cornu ammonis. In one patient, upper quadrantanopia developed after the operation. Medication was unchanged in one and reduced in two (seizures relapsed in one patient after medication had been discontinued). Two patients were treated with rethermolesion. One of them suffered an early relapse and was treated again in the 1st year after SAH. The other patient was treated for persistent auras. Both are Engel IA and have been on medication since.

After SAH alone, patients' medication was successfully discontinued in three cases. In five patients, it had to be reinstituted for seizure relapse. Medication was reduced in 14 , changed in six, unchanged in 22 , and increased in three patients.

\section{Early complications}

There was no procedure-related mortality or severe permanent morbidity. The most serious complication encountered was a small intracerebral hematoma in the probe trajectory and small hemocephalus in the occipital horn of the lateral ventricle, which caused obstructive hydrocephalus after a sudden head movement. This required a temporary placement of ventricular drainage and left no sequelae. In one patient, transitory anomia occurred. This was caused by a small cortical contusion in the entry point of the probe and resolved spontaneously. We noted upper quadrantanopia in one patient. Two small clinically silent hematomas in the trajectory were found during routine early MRI controls. In one patient, a small, clinically silent, subdural hematoma was found. All these hematomas resolved without treatment. There were two cases of meningitis, which were successfully treated with antibiotics. In one patient it was purulent and in the other aseptic. We subsequently initiated the application of a bolus of antibiotics before treatment and found no cases of meningitis thereafter. Four patients had headaches and upper meningeal syndrome. In these patients, we evaluated the cerebrospinal fluid and found no signs of inflammation. In another four patients with similar findings, we did not perform cerebrospinal fluid evaluation. All these patients were treated by common painkillers and became asymptomatic within 5 days. After that we concentrated on more rigorous trajectory planning to avoid lesions to the ependymal surface of the temporal horn of the lateral ventricle and did not subsequently find these complications. In one patient with hereditary coagulopathy, pulmonary embolism occurred and was treated with thrombolysis with no permanent sequelae. In two patients, a small fragment of the probe was left in the brain after operation. It was asymptomatic but precluded MRI controls. We noted no complications, or only slight headaches, in 44 patients. There certainly is a learning curve as the rate of complications tends to reduce with increasing experience.

\section{Late complications}

Three patients in our group died. Death could be attributed to epilepsy in one patient, who was seizure-free. He was postoperatively hospitalized for depression and committed suicide during the 3rd year after SAH. One patient died tragically after an accident during the second postoperative year, and the other from extracranial cancer during her third postoperative year.

One patient developed postictal psychosis and depression. In three patients, preexisting organic personality disorders worsened postoperatively. One patient was treated for postinterictal mania following antiepileptic drug discontinuation. This did not recur after treatment was reinstituted, but seizure control could not be achieved thereafter. One patient had refractory MTLE due to nondiagnosed limbic encephalitis and developed interictal psychosis. In one patient, psychogenic nonepileptic seizures and psychogenic amnesia manifested and were verified during subsequent video-electroencephalogram monitoring.

\section{Neuropsychological outcome}

Thirty-one patients with pre- and postoperative neuropsychological evaluation were available for analysis. ${ }^{28}$ In the group as a whole there were no statistically reliable changes in global, verbal, and visual memory quotient $(P=0.635$, 0.318 , and 0.855 , respectively) and all other memory subtests. There was a small, but statistically significant, improvement in verbal, performance and global intellectual performance ( $P=0.001,0.002$, and 0.004 , respectively). Patients showed significant improvement in the information $(P=0.028)$, vocabulary $(P=0.018)$, and object assembly $(P<0.0001)$ subtests.

At an individual level, our results are presented in Table 2. Three $(10 \%)$ patients showed significant improvement in global memory performance, one (4\%) patient deteriorated, and $25(86 \%)$ did not change. The patient who deteriorated in global memory was a right-handed, left-sided speech and 
A

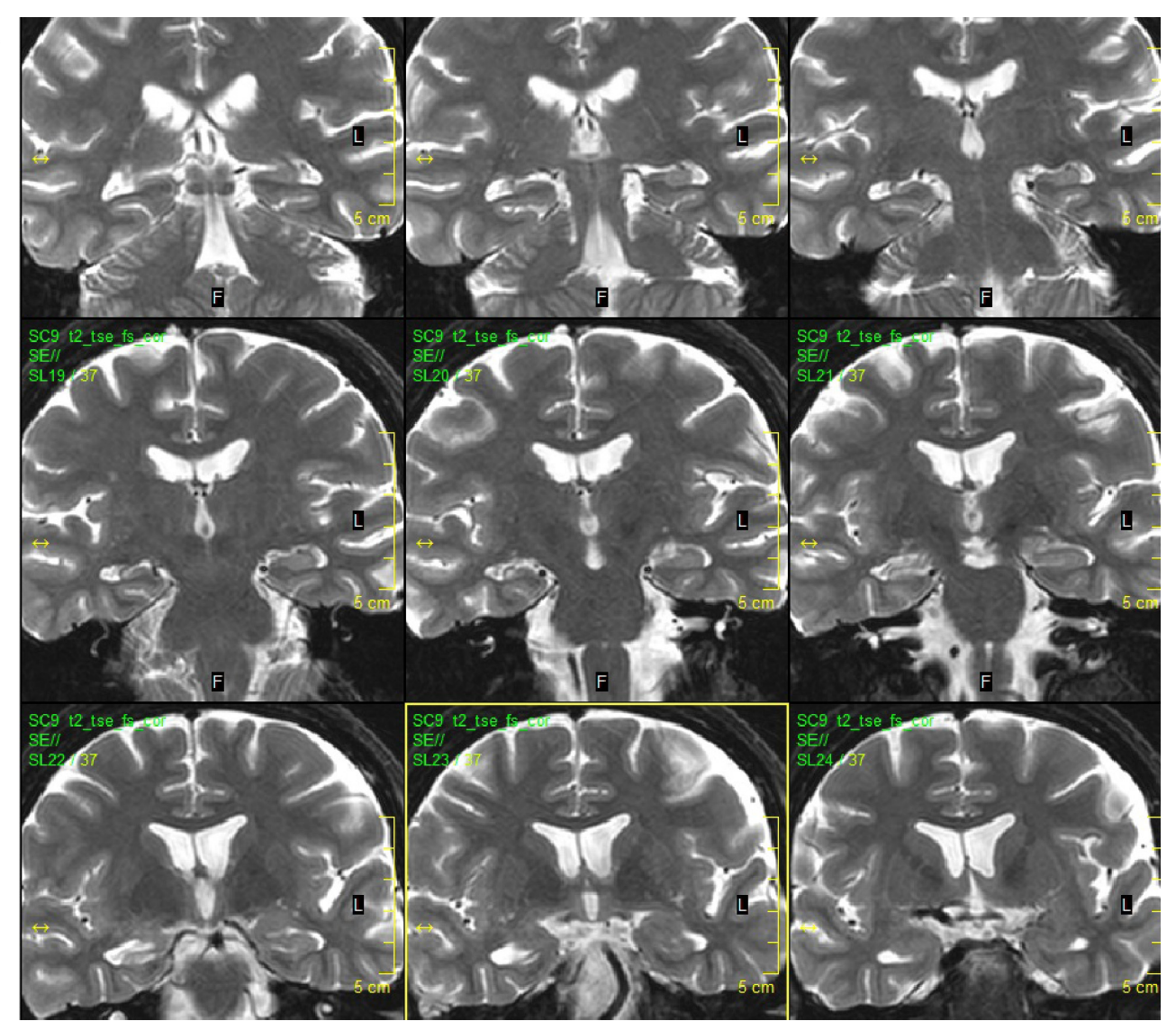

B

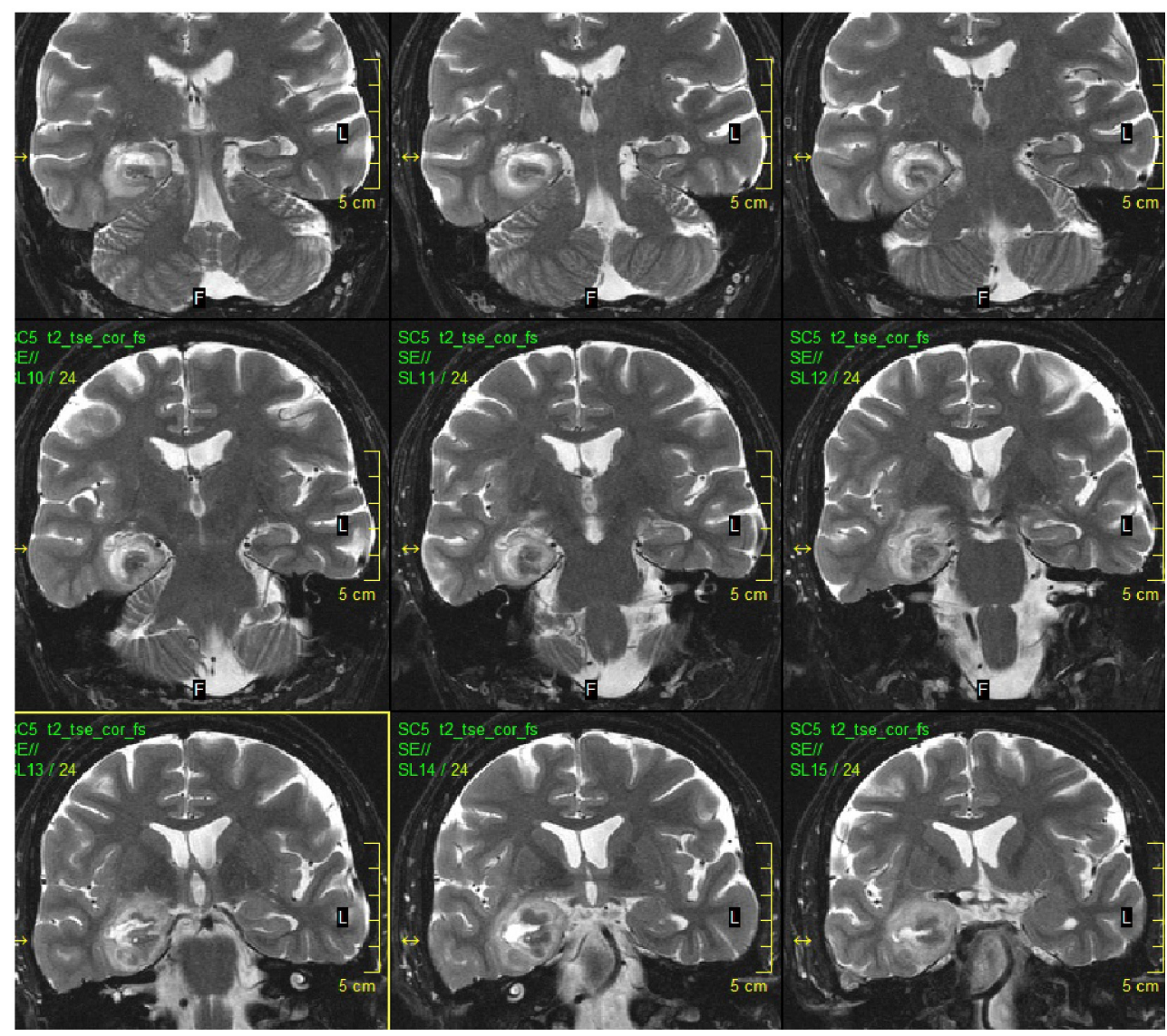




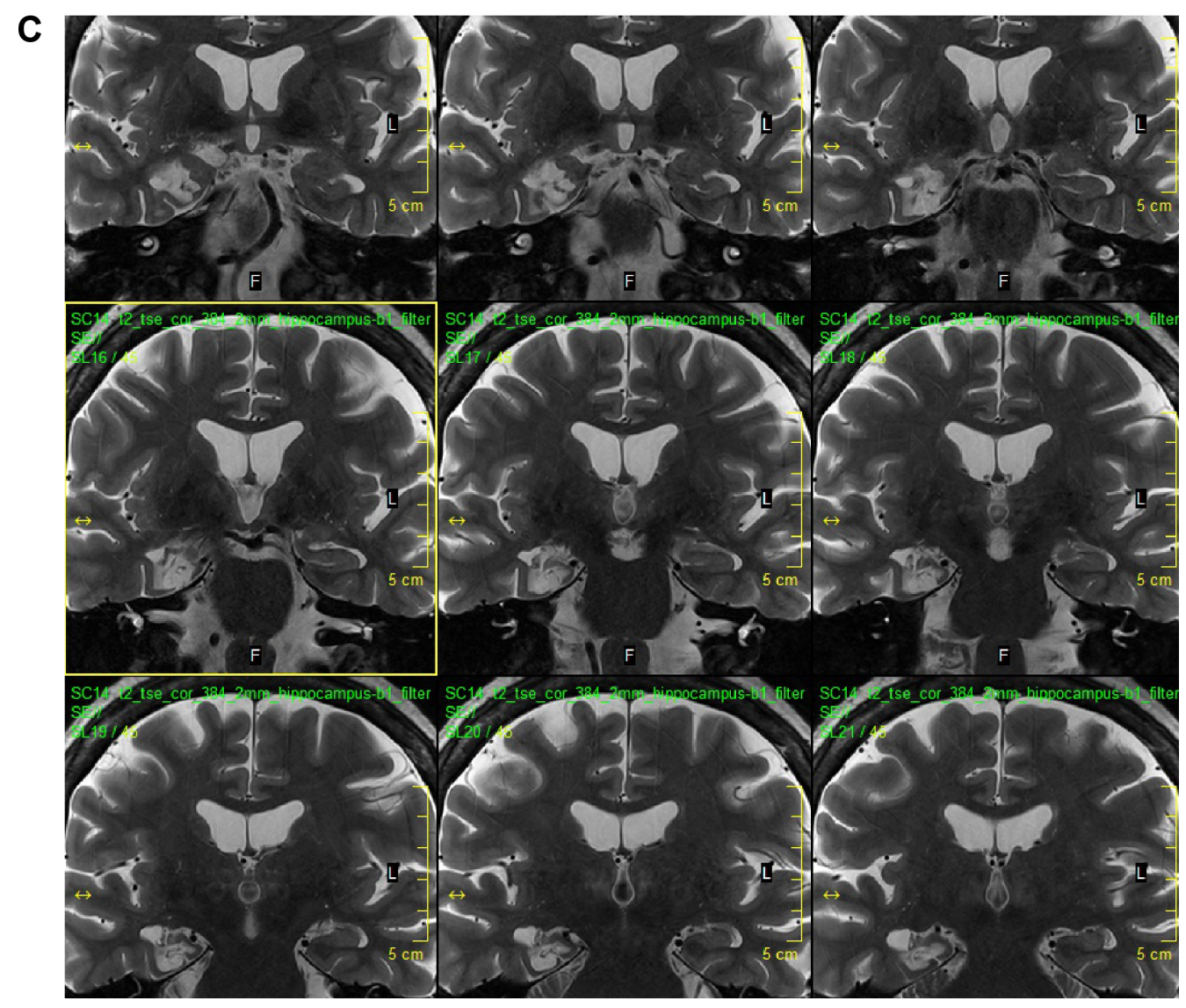

Figure 3 MRI before $(\mathbf{A})$, I week $(\mathbf{B})$, and I year $(\mathbf{C})$ after $\mathrm{SAH}$.

Abbreviations: MRI, magnetic resonance imaging; SAH, stereotactic radiofrequency amygdalohippocampectomy.

memory dominant person, operated on her left. She did not have HS and was not rendered seizure-free (Engel IIIB). All those who improved in global memory had clear HS, were operated on the nondominant side, and their postoperative course was favorable (Engel IC, IA, and IB, respectively). One patient $(3 \%)$ demonstrated reliable improvement and none showed deterioration in their verbal memory performance, while 28 (97\%) were unchanged. In visual memory performance, there was one patient with reliable deterioration, none improved, and 29 (97\%) of patients were unchanged. Interestingly, the patient who showed significant deterioration in visual memory was a right-handed person with left-sided speech and memory dominance, left-sided HS, and was operated on the left side. He was Engel class IB. Preoperative memory performance of both deteriorated patients was above average.

Five (17\%) patients improved in global intellectual performance, and $83 \%$ of patients remained unchanged. Verbal intelligence quotient improved in four (14\%) patients and performance intelligence quotient in four (13\%) patients; no deterioration was found. Patients with intellectual gain mostly had favorable seizure outcome. Sex, age of onset,

Table I Engel's classification during follow-up

\begin{tabular}{llllll}
\hline & Engel I & Engel IA & Engel II & Engel III & Engel IV \\
\hline FU-I $(n=6 I)$ & $4 I(67,2)$ & $28(45,9)$ & $13(21,3)$ & $4(6,7)$ & $3(4,9)$ \\
FU-2 $(n=59)$ & $42(7 I, 2)$ & $23(39,0)$ & $10(17,0)$ & $5(8,5)$ & $2(3,3)$ \\
FU-3 $(n=55)$ & $42(76,4)$ & $18(32,9)$ & $7(12,7)$ & $3(7,3)$ & $2(3,6)$ \\
FU-4 $(n=47)$ & $36(76,6)$ & $16(34,0)$ & $6(12,7)$ & $3(7,4)$ & $2(4,3)$ \\
FU-5 $(n=38)$ & $3 I(8 I, 5)$ & $18(47,4)$ & $3(10,3)$ & $1(3,4)$ & I $(2,6)$ \\
FU-6 $(n=29)$ & $25(86,2)$ & $12(4 I, 4)$ & $2(10,5)$ & & \\
FU-7 $(n=19)$ & $16(84,2)$ & $9(47,4)$ & $3(20,0)$ & & \\
FU-8 $(n=15)$ & $12(80,0)$ & $7(46,7)$ &
\end{tabular}

Note: Numbers in parentheses show percentages of patients classified by Engel's classification during follow-up I to 8 years after SAH.

Abbreviations: FU, follow-up; SAH, stereotactic radiofrequency amygdalohippocampectomy. 
Table 2 Neuropsychological outcome on a group and individual level

\begin{tabular}{|c|c|c|c|c|c|c|c|c|}
\hline & $\begin{array}{l}\text { Preoperative } \\
\text { n (SD) }\end{array}$ & $\begin{array}{l}\text { I-year FU } \\
\text { n (SD) }\end{array}$ & Mean change & Reliability & Significance & $\begin{array}{l}\text { Improvement } \\
\text { n (\%) }\end{array}$ & $\begin{array}{l}\text { No change } \\
\text { n (\%) }\end{array}$ & $\begin{array}{l}\text { Deterioration } \\
\text { n (\%) }\end{array}$ \\
\hline \multicolumn{9}{|c|}{ WMS-R } \\
\hline FS-MQ & 92.7 (I7.7) & $93.8(17.3)$ & -1.13 & 0.81 & 0.635 & $3(10)$ & $25(86)$ & I (4) \\
\hline VMQ & $90.0(19.1)$ & $92.5(17.9)$ & -2.50 & 0.77 & 0.318 & I (3) & $28(97)$ & $0(0)$ \\
\hline PMQ & $97.8(15.4)$ & $98.2(11.9)$ & -0.39 & 0.70 & 0.855 & $0(0)$ & $29(97)$ & I (3) \\
\hline \multicolumn{9}{|c|}{ WAIS-R } \\
\hline FS-IQ & $89.8(12.9)$ & $94.5(14.5)$ & -3.69 & 0.94 & 0.001 & $5(17)$ & $24(83)$ & $0(0)$ \\
\hline VIQ & $90.9(12.7)$ & $93.5(14.7)$ & -2.62 & 0.96 & 0.002 & $4(14)$ & $25(86)$ & $0(0)$ \\
\hline PIQ & $89.9(13.8)$ & $94.2(14.4)$ & -4.33 & 0.90 & 0.004 & $4(13)$ & $26(87)$ & $0(0)$ \\
\hline
\end{tabular}

Abbreviations: FS-IQ, full scale intelligence quotient; FS-MQ, full scale memory quotient; FU, follow-up; PIQ, performance intelligence quotient; PMQ, performance memory quotient; VIQ, visual intelligence quotient; VMQ, visual memory quotient; WAIS-R, Wechsler Adult Intelligence Scale-Revised; WMS-R, Wechsler Memory Scale-Revised.

presence of risk factors, seizure frequency, and epilepsy duration did not differ in those with significant memory or intellectual changes and mean group values.

\section{Volumetric results}

In 33 patients treated by SAH (23 patients) and microsurgical AHE (ten patients), we compared reduction in the volumes of the amygdala and hippocampus. ${ }^{30}$ In the SAH group, we found that amygdalar and hippocampal volumes were reduced by $58.0 \%$ and $55.2 \%$, respectively. In subjects treated by AHE these figures were $53.1 \%$ and $83.5 \%$, respectively. Thus, SAH leads to a similar reduction of the amygdalar volume but to a significantly lower reduction of the hippocampal volume than AHE.

In a group of 18 patients treated with $\mathrm{SAH}$, we found that seizure outcome was related to the extent of volume reduction. ${ }^{25}$ One year after the procedure, Engel's class I was achieved in $77 \%$ and $82 \%$ of those in whom hippocampal and amygdalar volumes were reduced by $\geq 50 \%$, respectively. In patients in whom volumes of both structures were reduced by $\geq 50 \%$, Engel's class IA was found in $89 \%$. In contrast, $56 \%$ of patients in whom both structures were not reduced by more than half reached Engel's class I. Surprisingly, there was no significant correlation of the clinical outcome to perirhinal and entorhinal cortex volume reductions in a group of 26 patients. ${ }^{31}$

We tested the relationship between hippocampal volume reduction and changes in cognitive performance after the operation separately for patients with surgery on the language-dominant hemisphere. Larger reductions were related to greater improvements in visual memory quotient in patients with surgery in the language-dominant hemisphere. No other relationships between hippocampal volume reduction and neuropsychological outcomes were significant, either at the individual level or in the group of patients as a whole. ${ }^{32}$

\section{$\mathrm{SAH}$ and open surgery}

We compared microsurgical AMTR and SAH with respect to seizure outcomes and complications in a group of 75 MTLE patients who were diagnosed at our institution. ${ }^{33}$ Forty-one of them were treated by SAH (eleven right-sided, 30 left-sided) and 34 by AMTR (21 right-sided, 13 left-sided). SAH and AMTR seizure control were comparable (Engel I in 75.6\% and $76.5 \% 2$ years after surgery and $79.3 \%$ and $76.5 \% 5$ years after procedures, respectively). The overall rate of surgical nonsilent complications without permanent neurological deficit after AMTR was $11.8 \%$ (one case of meningitis; three infarctions, two dysphasias, and one hemiparesis). All complications were transient and no persistent neurological deficit lasting more than 1 year after operation was found. The overall rate of silent infarctions found on follow-up MRI after AMTR was $8.8 \%$. The rate of clinically manifest complications after SAH was $4.9 \%$ (one meningitis requiring antibiotic therapy and one case of transitory anomia, probably caused by a small cortical hematoma in the entry point of the probe, which resolved spontaneously).

\section{Discussion}

Up to now stereotactic treatment was thought to be dedicated to drug-resistant epileptic patients for whom conventional resection surgery is risky or contraindicated..$^{34}$ In our group of patients, we demonstrated that SAH is a feasible and safe treatment modality. Our modification of SAH is the first stereotactic epilepsy surgery to yield seizure outcomes comparable to those of conventional epilepsy surgery in patients with MTLE. ${ }^{35}$ However, in some highly selected series, seizure-free outcome is as high as $90 \%{ }^{36}$ It should be stressed that our patients formed a highly heterogenous group. There were various degrees of certainty of the mesiotemporal localization of the epileptogenic zone based on varying degrees of congruence between preoperative diagnostic methods. Besides HS, some patients harbored another 
potentially epileptogenic lesion. There were nonlesional cases and patients with a burned-out hippocampus. Furthermore, patients were also indicated for treatment from two other institutions. Referring physicians could have other views and may consider SAH a palliative procedure in patients, with incomplete congruence of results from the preoperative work-up or judge them not eligible for conventional surgery for other reasons (eg, age, comorbidity, etc).

One of the reasons in favor of SAH is that the extent of stereotactic treatment could be more precisely planned than in conventional surgery. The literature documents that the extent of the resection planned and that ultimately performed need not necessarily correlate in conventional epilepsy surgery. ${ }^{7}$ On the other hand, incomplete destruction may be the cause of treatment failure or seizure recurrence after antiepileptic drugs discontinuation in some patients. It is not clear whether antiepileptic drug discontinuation could be more easily achieved in SAH or open surgery. However, it seems reasonable to start with minimally invasive treatment when the chances of a cure are comparable to more extensive procedures. In cases where treatment fails due to an individual anatomic situation which obviates complete coverage of the target structures or due to a more extended epileptogenic zone, subsequent epilepsy surgery can still be safely and effectively performed.

We speculate that extensive coverage of target structures with their incomplete destruction could be as effective as their complete lesion. It is debatable as to whether $\mathrm{SAH}$ would be effective in cases of dual pathology (for example, mesiotemporal focal cortical dysplasia associated with HS) in which complete coverage of both lesions is possible. In cases where part of the epileptogenic area lies outside the mesial temporal lobe structures, optimal outcomes can certainly only be achieved when mesial and neocortical structures are removed by open surgery. Hopefully, prospective evaluation of more homogenous groups of patients with MTLE/HS will bring up even better results.

There might be concerns about our list of complications. We should stress that most early complications are transitory or minor. Reports on open surgery do not usually mention signs such as headache and stiff neck. However, we must admit that the rate of mild visual field defects could be higher as we only evaluated the perimeter as part of the neurological evaluation, and formal visual field examination was only performed on patients who had suspected visual field defects.

In the literature, major complications after temporal resections are reported in about $4.1 \%$ of cases, and the occurrence of minor complications is reported in about $11.5 \%$ of cases. ${ }^{37}$ The rate of silent postoperative infarctions in our open surgery group was surprising as most studies do not report any silent infarcts. Unfortunately, not all reports on open surgery use MRI control evaluation. We suggest that infarctions, although clinically silent, are the unwanted result of epilepsy surgery and are underestimated in the literature. In one study, 15 patients were resected and underwent postoperative MRI. In three patients infarction was found, but only one was clinically symptomatic. ${ }^{38}$ Surgery for MTLE is associated with specific cognitive risks. ${ }^{39}$ The only way to improve neuropsychological results is by minimizing the destruction of both nonepileptic and epileptic, yet still functional, mesial temporal structures. Stereotactic neurosurgery represents a move towards greater selectivity, with minimal damage to the brain tissue on the access route to the target structure. In comparison to other stereotactic methods, our occipital approach offers more complete coverage of the amygdala and hippocampus, makes lesioning of the PHG possible, and minimizes white matter damage. The destruction of the target structures is only about $50 \%$.

At the group level we demonstrated slight intellectual improvement and no memory change. It is noteworthy that, at an individual level, we found more improved than deteriorated patients in both the memory and intelligence domains. We would like to stress that there was no case where verbal memory worsened, even though left-sided operations prevailed. Postoperative neuropsychological improvement has been interpreted as a practice effect by some authors. Although we used the reliable change indexes method to eliminate it, we cannot exclude the possibility that this effect played some role.

We hypothesize that positive neuropsychological results were achieved by the incomplete destruction of target structures, sparing the lateral temporal neocortex. $\mathrm{SAH}$ enables the longitudinal intrinsic hippocampal connections to be targeted, providing relative protection to circuits perpendicular to the long axis of the hippocampus. This could lead to a reduction in the transmission of epileptic discharges to other structures. In this respect, SAH remotely resembles multiple hippocampal transections in which favorable neuropsychological outcomes were also reported. ${ }^{40} \mathrm{We}$ are aware that these other structures targeted by SAH must be changed by gliosis and atrophy, and we are as yet ignorant of their ability to reorganize and support cognitive functions. However, we are also unaware of their epileptogenic potential.

As HS was only found as an epileptogenic substrate in a minority of surgically treated patients in some series, the 
lack of material for histopathological evaluation is certainly a disadvantage of $\mathrm{SAH} .{ }^{41}$

Our study has several limitations: 1) there is no control group of patients treated by standard epilepsy surgery methods; 2) the main drawback of our series is the inhomogeneity of the patient population. There are unequal numbers of patients treated on the left and right sides and this is certainly due to a selection bias; 3) we cannot answer the question as to whether our patients could achieve a seizure-free outcome from medication as we suggested antiepileptic drug discontinuation in a minority of our patients; 4) we cannot assess the degree of tissue destruction in our successfully treated patients as there are no histopathological specimens available; 5) the neuropsychological evaluation battery is limited, and some cognitive functions have not been sampled; 6) results of pre- and postoperative cognitive functions evaluation were only available in a subset of the treated patients; and 7) this study shows a single center experience.

\section{Conclusion}

$\mathrm{SAH}$ is a promising method in the treatment of intractable patients with MTLE. Our experience provides preliminary evidence for good long-term seizure control comparable to open surgery. The results suggest that SAH could be superior to open surgery in terms of its neurocognitive outcomes. SAH could be an alternative therapy for MTLE. Further randomized prospective multicenter studies are warranted to prove this concept.

\section{Acknowledgments}

Supported by the Ministry of Health, Czech Republic conceptual development of a research organization (Nemocnice Na Homolce - NNH, 00023884). We are indebted to Petr Marusič, MD, $\mathrm{PhD}$ and Tomáš Nežádal, $\mathrm{MD}, \mathrm{PhD}$ for providing follow-up information about their patients and Mrs Carey Vosecká, MA, LLB for help with the English editing.

\section{Disclosure}

The authors report no conflicts of interest in this work.

\section{References}

1. Malmgren K, Thom M. Hippocampal sclerosis - origins and imaging. Epilepsia. 2012;53(Suppl 4):19-33.

2. Engel J, Wiebe S, French J, et al. Practice parameter: temporal lobe and localized neocortical resections for epilepsy. Epilepsia. 2003; 44(6):741-751.

3. Spencer DD, Spencer SS, Mattson RH, Williamson PD, Novelly RA. Access to the posterior medial temporal lobe structures in the surgical treatment of temporal lobe epilepsy. Neurosurgery. 1984;15(5):667-671.
4. Yaşargil MG, Krayenbühl N, Roth P, Hsu SP, Yaşargil DC. The selective amygdalohippocampectomy for intractable temporal limbic seizures. J Neurosurg. 2010;112(1):168-185.

5. Olivier A. Transcortical selective amygdalohippocampectomy in temporal lobe epilepsy. Can J Neurol Sci. 2000;27(Suppl 1):S68-S76; discussion S92-S96.

6. Ramey WL, Martirosyan NL, Lieu CM, Hasham HA, Lemole GM, Weinand ME. Current management and surgical outcomes of medically intractable epilepsy. Clin Neurol Neurosurg. 2013;115(12): 2411-2418.

7. Schramm J. Temporal lobe epilepsy surgery and the quest for optimal extent of resection: a review. Epilepsia. 2008;49(8):1296-1307.

8. Hamberger MJ, Drake EB. Cognitive functioning following epilepsy surgery. Curr Neurol Neurosci Rep. 2006;6(4):319-326.

9. Dulay MF, Busch RM. Prediction of neuropsychological outcome after resection of temporal and extratemporal seizure foci. Neurosurg Focus. 2012;32(3):E4.

10. Baxendale S, Thompson PJ, Duncan JS. Improvements in memory function following anterior temporal lobe resection for epilepsy. Neurology. 2008;71(17):1319-1325.

11. Schaller C, Jung A, Clusmann H, Schramm J, Meyer B. Rate of vasospasm following the transsylvian versus transcortical approach for selective amygdalohippocampectomy. Neurol Res. 2004;26(6):666-670.

12. Koch-Stoecker S, Schmitz B, Kanner AM. Treatment of postsurgical psychiatric complications. Epilepsia. 2013;54(Suppl 1):46-52.

13. Mengesha T, Abu-Ata M, Haas KF, et al. Visual field defects after selective amygdalohippocampectomy and standard temporal lobectomy. J Neuroophthalmol. 2009;29(3):208-213.

14. Georgiadis I, Kapsalaki EZ, Fountas KN. Temporal lobe resective surgery for medically intractable epilepsy: a review of complications and side effects. Epilepsy Res Treat. 2013;2013:752195.

15. Polkey C. Surgical alternatives for the treatment of temporal lobe epilepsy. Br J Neurosurg. 1988;2(2):143-152.

16. Bouchard G, Kim YK, Umbach W. Stereotaxic methods in different forms of epilepsy. Confin Neurol. 1975;37(1-3):232-238.

17. Mempel E, Witkiewicz B, Stadnicki R, et al. The effect of medial amygdalotomy and anterior hippocampotomy on behavior and seizures in epileptic patients. Acta Neurochir Suppl (Wien). 1980;30:161-167.

18. Talairach J, Szikla G. [Amygdalo-hippocampal partial destruction by yttrium-90 in the treatment of certain epilepsies of rhinencephalic manifestation]. Neurochirurgie. 1965;11(3):233-240. French.

19. Vladyka V. Tactics in surgical treatment of epilepsy and its realization in cases of temporal epilepsy. Ces Slov Neurol Neurochir. 1978;41(74):95-106.

20. Kratimenos GP, Pell MF, Thomas DG, Shorvon SD, Fish DR, Smith SJ. Open stereotactic selective amygdalo-hippocampectomy for drug resistant epilepsy. Acta Neurochir (Wien). 1992;116(2-4):150-154.

21. Patil AA, Andrews R, Torkelson R. Stereotactic volumetric radiofrequency lesioning of intracranial structures for control of intractable seizures. Stereotact Funct Neurosurg. 1995;64(3):123-133.

22. Blume WT, Parrent AG, Kaibara M. Stereotactic amygdalohippocampectomy and mesial temporal spikes. Epilepsia. 1997;38(8):930-936.

23. Parrent AG, Blume WT. Stereotactic amygdalohippocampotomy for the treatment of medial temporal lobe epilepsy. Epilepsia. 1999; 40(10):1408-1416.

24. Vojtěch Z, Malíková H, Krámská L, et al. Long-term seizure outcome after stereotactic amygdalohippocampectomy. Acta Neurochir (Wien). 2014;156(8):1529-1537.

25. Malíková H, Vojtěch Z, Liščák R, et al. Stereotactic radiofrequency amygdalohippocampectomy for the treatment of mesial temporal lobe epilepsy: correlation of MRI with clinical seizure outcome. Epilepsy Res. 2009;83(2-3):235-242.

26. Watson C, Jack CR Jr, Cendes F. Volumetric magnetic resonance imaging. Clinical applications and contributions to the understanding of temporal lobe epilepsy. Arch Neurol. 1997;54(12):1521-1531.

27. MacMaster FP, Kusumakar V. Hippocampal volume in early onset depression. BMC Med. 2004;2:2. 
28. Vojtěch Z, Krámská L, Malíková H, et al. Cognitive outcome after stereotactic amygdalohippocampectomy. Seizure. 2012;21(5):327-333.

29. Liščák R, Malíková H, Kalina $M$, et al. Stereotactic radiofrequency amygdalohippocampectomy in the treatment of mesial temporal lobe epilepsy. Acta Neurochir (Wien). 2010;152(8):1291-1298.

30. Malíková H, Vojtěch Z, Liščák R, et al. Microsurgical and stereotactic radiofrequency amygdalohippocampectomy for the treatment of mesial temporal lobe epilepsy: different volume reduction, similar clinical seizure control. Stereotact Funct Neurosurg. 2010;88(1):42-50.

31. Malíková H, Liščák R, Vojtěch Z, et al. Stereotactic radiofrequency amygdalohippocampectomy: does reduction of entorhinal and perirhinal cortices influence good clinical seizure outcome? Epilepsia. 2011;52(5):932-940.

32. Malíková H, Krámská L, Liščák R, et al. Stereotactic radiofrequency amygdalohippocampectomy for the treatment of temporal lobe epilepsy: do good neuropsychological and seizure outcomes correlate with hippocampal volume reduction? Epilepsy Res. 2012;102(1-2):34-44.

33. Maliková H, Krámská L, Vojtěch Z, et al. Different surgical approaches for mesial temporal epilepsy: resection extent, seizure, and neuropsychological outcomes. Stereotact Funct Neurosurg. 2014;92(6):372-380.

34. Guénot M, Isnard J, Catenoix H, Mauguière F, Sindou M. SEEG-guided RF-thermocoagulation of epileptic foci: a therapeutic alternative for drug-resistant non-operable partial epilepsies. Adv Tech Stand Neurosurg. 2011;36:61-78.
35. Téllez-Zenteno JF, Dhar R, Wiebe S. Long-term seizure outcomes following epilepsy surgery: a systematic review and meta-analysis. Brain. 2005;128(Pt 5):1188-1198.

36. Elliott RE, Bollo RJ, Berliner JL, et al. Anterior temporal lobectomy with amygdalohippocampectomy for mesial temporal sclerosis: predictors of long-term seizure control. J Neurosurg. 2013;119(2):261-272.

37. Hader WJ, Téllez-Zenteno J, Metcalfe A, et al. Complications of epilepsy surgery: a systematic review of focal surgical resections and invasive EEG monitoring. Epilepsia. 2013;54(5):840-847.

38. Engel J, McDermott MP, Wiebe S, et al; Early Randomized Surgical Trial (ERSET) Study Group. Early surgical therapy for drugresistant temporal lobe epilepsy: a randomized trial. JAMA. 2012; 307(9):922-930.

39. Sherman EM, Wiebe S, Fay-McClymont TB, et al. Neuropsychological outcomes after epilepsy surgery: systematic review and pooled estimates. Epilepsia. 2011;52(5):857-869.

40. Shimizu H, Kawai K, Sunaga S, Sugano H, Yamada T. Hippocampal transection for treatment of left temporal lobe epilepsy with preservation of verbal memory. J Clin Neurosci. 2006;13(3):322-328.

41. Tassi L, Meroni A, Deleo F, et al. Temporal lobe epilepsy: neuropathological and clinical correlations in 243 surgically treated patients. Epileptic Disord. 2009;11(4):281-292. 


\section{Supplementary materials}

Table SI

\begin{tabular}{|c|c|c|c|c|c|c|c|c|c|c|c|}
\hline Pt no & Sex & Born & Risk factors & Start & Duration & Age & MRI & PET & Scalp/invas & Side & Date \\
\hline I & $M$ & 1956 & $\mathrm{PT} / \mathrm{FS}$ & II & 37 & 48 & $\mathrm{HS} \sin$ & $T \sin$ & $\sin /-$ & $\sin$ & $2004-04-28$ \\
\hline 2 & $M$ & 1946 & None & 40 & 18 & 58 & HS sin, vs biL & $T d x$ & $d x / \sin$ & $\sin$ & $2004-05-12$ \\
\hline 3 & $M$ & 1944 & None & 31 & 29 & 60 & N/A & $T \sin$ & $\sin /-$ & $\sin$ & $2004-06-02$ \\
\hline 4 & $M$ & 1970 & None & 10 & 24 & 34 & $\mathrm{HS} d \mathrm{x}$ & $T d x$ & $\mathrm{dx} /-$ & $d x$ & 2004-09-08 \\
\hline 5 & $\mathrm{~F}$ & 1961 & None & 15 & 28 & 43 & $H S \sin$ & $T \sin$ & $\sin /-$ & $\sin$ & $2004-12-15$ \\
\hline 6 & $\mathrm{~F}$ & 1977 & FS & 5 & 23 & 28 & $H S \sin$ & $T \sin$ & $\sin /-$ & $\sin$ & 2005-06-0I \\
\hline 7 & $M$ & 1969 & FS & 9 & 27 & 36 & HS dx & $\mathrm{Tdx}$ & $d x /-$ & $d x$ & $2005-06-02$ \\
\hline 8 & $F$ & 1940 & None & 4 & 61 & 65 & $\mathrm{HS} \sin$ & $T \sin$ & $\sin /-$ & $\sin$ & $2005-06-08$ \\
\hline 9 & $M$ & 1967 & Chemotherapy & 14 & 24 & 38 & neg & $T \sin$ & $\sin /-$ & $\sin$ & $2005-07-27$ \\
\hline 10 & $M$ & 1976 & TBI & 14 & 15 & 29 & HS sin+ dyspl P & $T \sin$ & $\sin /-$ & $\sin$ & $2005-07-28$ \\
\hline II & $M$ & 1955 & None & 3 & 47 & 50 & $H S \sin$ & $T \sin$ & $\sin /-$ & $\sin$ & $2005-10-26$ \\
\hline 12 & $M$ & 1978 & $\mathrm{PT} / \mathrm{FS}$ & 6 & 21 & 27 & $H S \sin$ & $T \sin$ & $\sin /-$ & $\sin$ & $2005-10-27$ \\
\hline 13 & $F$ & 1973 & None & 15 & 17 & 32 & neg & $T \sin$ & $\sin /-$ & $\sin$ & $2005-12-14$ \\
\hline 14 & $\mathrm{~F}$ & 1985 & PT/FS & 17 & 3 & 20 & HS dx+- & $\mathrm{Tdx}+-$ & $d x /+$ & $d x$ & $2005-12-15$ \\
\hline 15 & $\mathrm{~F}$ & 1977 & FS & 20 & 9 & 29 & HS dx & $T d x$ & $d x /-$ & $d x$ & 2006-0I-05 \\
\hline 16 & $M$ & 1956 & $\mathrm{PT} / \mathrm{FS}$ & 19 & 31 & 50 & $\mathrm{HS} d \mathrm{x}$ & $T d x$ & $d x /-$ & $\mathrm{dx}$ & $2006-02-15$ \\
\hline 17 & $\mathrm{~F}$ & 1978 & FS & 12 & 16 & 28 & $\mathrm{HS} \sin$ & $T \sin$ & bilat/sin & $\sin$ & 2006-03-0I \\
\hline 18 & $F$ & 1970 & ME/FS & 1 & 35 & 36 & $\mathrm{HS} \sin$ & $T \sin$ & $\sin /-$ & $\sin$ & $2006-06-15$ \\
\hline 19 & $\mathrm{~F}$ & 1989 & FS & II & 6 & 17 & $\mathrm{HS} \sin$ & $T \sin$ & $\sin /-$ & $\sin$ & 2006-06-29 \\
\hline 20 & $M$ & 1970 & ME/FS & 10 & 26 & 36 & $\mathrm{HS} \sin$ & $T \sin$ & $\sin /-$ & $\sin$ & $2006-08-02$ \\
\hline 21 & $F$ & 1953 & $\mathrm{TBI}$ & 44 & 10 & 54 & neg & $T d x$ & $d x /-$ & $d x$ & $2007-01-24$ \\
\hline 22 & $M$ & 1978 & ME & 4 & 25 & 29 & $\mathrm{HS} \sin$ & $T \sin$ & $\sin /-$ & $\sin$ & $2007-0 \mathrm{I}-25$ \\
\hline 23 & $F$ & 1961 & ME & 22 & 24 & 46 & $H S \sin$ & $T \sin$ & $\sin /-$ & $\sin$ & 2007-03-15 \\
\hline 24 & $F$ & 1986 & FS & 2 & 19 & 21 & $H S \sin$ & $T \sin$ & $\sin /-$ & $\sin$ & 2007-04-18 \\
\hline 25 & $M$ & 1960 & ME & 37 & 10 & 47 & $\mathrm{HS} \sin$ & $T \sin$ & $\sin /-$ & $\sin$ & $2007-06-06$ \\
\hline 26 & $M$ & 1974 & None & 13 & 20 & 33 & $\mathrm{HS} \sin$ & $T \sin$ & $\sin /-$ & $\sin$ & $2007-06-20$ \\
\hline 27 & $M$ & 1961 & None & 10 & 36 & 46 & $\mathrm{HS} \sin$ & $T \sin$ & $\sin /-$ & $\sin$ & 2007-08-29 \\
\hline 28 & $M$ & 1956 & None & 17 & 34 & 51 & $\mathrm{HS} \sin$ & $T \sin$ & $\sin /-$ & $\sin$ & 2007-09-05 \\
\hline 29 & $M$ & 1952 & None & 4 & 51 & 55 & $H S \sin$ & $T \sin$ & $\sin /-$ & $\sin$ & 2007-10-24 \\
\hline 30 & $F$ & 1973 & FS & 25 & 9 & 34 & HS dx & $T d x$ & $d x /-$ & $d x$ & 2007-II-08 \\
\hline 31 & $F$ & 1973 & None & II & 23 & 34 & $H S \sin$ & $T \sin$ & $\sin /-$ & $\sin$ & 2007-II-07 \\
\hline 32 & $M$ & 1932 & FS & 1.5 & 73 & 75 & HS sin, atrophy & $T \sin$ & $\sin / \sin$ & $\sin$ & 2007-II-07 \\
\hline 33 & $\mathrm{~F}$ & 1981 & ME & 20 & 6 & 26 & $\mathrm{HS} \sin$ & $T \sin$ & bilat/- & $\sin$ & $2007-12-06$ \\
\hline 34 & $\mathrm{~F}$ & I94I & None & 41 & 26 & 67 & $\mathrm{HS} \sin$ & $T \sin$ & $\sin /-$ & $d x$ & 2007-06-2I \\
\hline 35 & $M$ & 1967 & None & 30 & 11 & 41 & HS dx & $T d x$ & bilat/dx & $d x$ & $2008-01-16$ \\
\hline 36 & M & 1948 & None & 32 & 28 & 60 & HS bilat & $T \sin$ & $\sin /-$ & $\sin$ & $2008-01-30$ \\
\hline 37 & $M$ & 1982 & PT/ME/FS & 13 & 13 & 26 & $H S \sin$ & NP & $\sin /-$ & $\sin$ & $2008-02-27$ \\
\hline 38 & $M$ & 1962 & ME & 10 & 36 & 46 & $\mathrm{HS} \sin$ & $T \sin$ & $\sin /-$ & $\sin$ & 2008-04-09 \\
\hline 39 & $F$ & 1987 & FS & 4 & 17 & 21 & HS bilat & negat & $\sin /-$ & $\sin$ & $2008-07-30$ \\
\hline 40 & $\mathrm{~F}$ & 1975 & FS & 5 & 28 & 33 & neg & $T \sin$ & $\sin /-$ & $\sin$ & 2008-07-31 \\
\hline 41 & $F$ & 1958 & None & 45 & 5 & 50 & $\mathrm{HS} d \mathrm{x}$ & $T d x$ & $d x /-$ & $d x$ & $2008-09-10$ \\
\hline 42 & $M$ & 1957 & FS & 35 & 16 & 51 & neg & $T d x$ & $d x /-$ & $d x$ & 2008-12-04 \\
\hline 43 & $M$ & 1966 & FS & 31 & 12 & 43 & $H S \sin$ & NP & $\sin /-$ & $\sin$ & $2008-12-10$ \\
\hline 44 & $\mathrm{~F}$ & 1976 & FS & 10 & 23 & 33 & $\mathrm{HS} \sin$ & $T \sin$ & $\sin /-$ & $\sin$ & 2009-04-09 \\
\hline 45 & $M$ & 1974 & None & 20 & 13 & 33 & HS dx & $T d x$ & $d x /-$ & $d x$ & 2009-06-09 \\
\hline 46 & $M$ & 1977 & FS & 5 & 27 & 32 & HS dx & negat & $\mathrm{dx} /-$ & $d x$ & $2009-06-18$ \\
\hline 47 & $F$ & 1976 & FS & 13 & 20 & 33 & $H S \sin$ & $T \sin$ & $d x / \sin$ & $\sin$ & 2009-0I-07 \\
\hline 48 & $F$ & 1948 & None & 30 & 31 & 61 & $\mathrm{HS} \sin$ & $T \sin$ & $\sin /-$ & $\sin$ & $2009-07-16$ \\
\hline 49 & $\mathrm{~F}$ & 1988 & None & 8 & 13 & 21 & $\mathrm{~N} / \mathrm{A}$ & $\mathrm{N} / \mathrm{A}$ & $d x /-$ & $d x$ & 2009-09-09 \\
\hline 50 & $M$ & 1979 & None & 18 & 12 & 30 & HS dx & $T d x$ & $d x /-$ & $d x$ & 2009-09-10 \\
\hline 51 & $M$ & 1964 & FS & 8 & 37 & 45 & $\mathrm{HS} \sin$ & $T \sin$ & $\sin /-$ & $\sin$ & 2009-09-17 \\
\hline 52 & $\mathrm{~F}$ & 1977 & ME & 13 & 19 & 32 & $\mathrm{HS} \sin$ & $T \sin$ & bilat & $\sin$ & 2009-10-08 \\
\hline
\end{tabular}

(Continued) 
Table SI (Continued)

\begin{tabular}{|c|c|c|c|c|c|c|c|c|c|c|c|}
\hline Pt no & Sex & Born & Risk factors & Start & Duration & Age & MRI & PET & Scalp/invas & Side & Date \\
\hline 53 & $M$ & 1975 & $\mathrm{PT} / \mathrm{nll} / \mathrm{str}$ & 4 & 31 & 35 & HS sin+ dyspl biO & $T \sin$ & $\sin /-$ & $\sin$ & $2009-12-10$ \\
\hline 54 & $\mathrm{~F}$ & $196 \mid$ & None & 10 & 39 & 49 & HS dx & $T d x$ & $\mathrm{dx} /-$ & $d x$ & $2010-03-24$ \\
\hline 55 & $\mathrm{~F}$ & 1946 & ME & 54 & 10 & 64 & HS dx & $\mathrm{Tdx}$ & $\mathrm{dx} /-$ & $d x$ & $2010-04-22$ \\
\hline 56 & M & 1972 & LE & 20 & 18 & 38 & $\mathrm{HS} \sin$ & $T \sin$ & $\sin /-$ & $\sin$ & $2010-05-27$ \\
\hline 57 & $\mathrm{~F}$ & 1980 & FS & 28 & 2 & 30 & neg & $T \sin$ & $\sin /-$ & $\sin$ & $2010-06-30$ \\
\hline 58 & $\mathrm{~F}$ & 1956 & ME & 12 & 42 & 54 & HS dx+ atrophy & $T d x$ & $\mathrm{dx} /-$ & $d x$ & $2010-09-0 \mid$ \\
\hline 59 & M & 1965 & FS & 16 & 29 & 45 & HS dx & $T d x$ & $\mathrm{dx} /-$ & $d x$ & $2010-09-08$ \\
\hline 60 & $F$ & $|96|$ & PT & 3 & 46 & 49 & $\mathrm{HS} d x+\mathrm{PVH}$ & negat & bilat/- & $d x$ & $2010-09-16$ \\
\hline 61 & $\mathrm{~F}$ & 1939 & ME/FS & 18 & 56 & 74 & $\mathrm{HS} \sin$ & NP & $\sin /-$ & $\sin$ & $2010-10-13$ \\
\hline
\end{tabular}

Note: Dates are presented as year-month-day. Patients with less than perfect agreement of preoperative results are set off in orange. Patients with another potentially epileptogenic lesion are blue. The patient with both conditions is green.

Abbreviations: bilat, bilateral; biO, bioccipital; dyspl, dysplasia; dx, right; FS, febrile seizures; invas, invasive; HS, hippocampal sclerosis; LE, limbic encephalitis; ME, meningoencephalitis; MRI, magnetic resonance imaging; N/A, not available; nll/str, optic nerve atrophy, strabism; NP, not performed; neg, negative (without pathological finding); PET, positron emission tomography; Pt no, patient number; PT, perinatal trauma; PVH, periventricular heterotopia; sin, left; $\mathrm{T}$, in the temporal lobe; TBI, traumatic brain injury.

\section{Table S2}

\begin{tabular}{|c|c|c|c|c|c|c|c|c|c|c|c|c|c|}
\hline Pt no & Early c & Late c & FU-I & FU-2 & FU-3 & FU-4 & FU-5 & FU-6 & FU-7 & FU-8 & Follow-up & Notes & Medication \\
\hline $\mathrm{I}$ & $M$ & & $\mathrm{IA}$ & IA & $\mathrm{IA}$ & IA & $\mathrm{IA}$ & ID & $\mathrm{IA}$ & IA & 8 & VNS-impl 2003, expl 2007 & $\mathrm{R}$ \\
\hline 2 & $\mathrm{DP}, \mathrm{CC}$ & $\mathrm{D}$ & IA & IA & suicide & & & & & & 2 & PA & N/A (U) \\
\hline 3 & $\mathrm{M}, \mathrm{PE}$ & & IA & IA & IA & IA & IA & IA & IA & IA & 8 & SAH in the 1970 s & $\mathrm{D}$ \\
\hline 4 & $\mathrm{SDH}$ & & IIB & IIB & IC & IB & IB & IB & IB & IA & 8 & & $\mathrm{R}$ \\
\hline 5 & $\mathrm{MS} / \mathrm{P}$ & & IB & IB & IB & IB & IB & $\mathrm{IB}$ & IB & IB & 8 & & $\mathrm{R}$ \\
\hline 6 & MS/P, QA & & IA & IA & ID & ID & $\mathrm{IA}$ & IA & IA & IB & 8 & & $\mathrm{U}(\mathrm{RR})$ \\
\hline 7 & $\mathrm{H}$ & & IIB & IC & IC & IC & IA & IA & IA & IA & 8 & & $\mathrm{R}$ \\
\hline 8 & $\mathrm{H}$ & & IA & IB & IB & IB & IB & IB & IB & IA & 8 & & $\mathrm{R}$ \\
\hline 9 & $\mathrm{MS} / \mathrm{P}$ & & IIB & IC & IIIB & IC & IC & IC & IC & IA & 8 & & $\mathrm{CH}$ \\
\hline 10 & $\mathrm{MS} / \mathrm{N}$ & & $\mathrm{IA}$ & IA & IA & $\mathrm{IA}$ & IA & IA & $\mathrm{IA}$ & IB & 8 & & $\mathrm{R}$ \\
\hline II & $\mathrm{H}$ & & $\mathrm{IA}$ & IA & IA & IA & IA & IA & IA & ID & 8 & PA & $\mathrm{R}$ \\
\hline 12 & $\mathrm{BP}$ & $\mathrm{D}, \mathrm{PIP}$ & $\mathrm{IA}$ & IA & $\| A$ & IIA & IIB & IIB & IIB & IA & 8 & & $U(R R)$ \\
\hline 13 & None & & IB & IB & IB & IB & IB & IB & IB & IIB & 8 & & $\mathrm{R}$ \\
\hline 14 & None & PNES & IIB & IIB & IC & IC & II B & IIB & IB & IB & 8 & & I \\
\hline 15 & $\mathrm{BP}$ & & IIB & IIA & IIB & IIB & IIB & IIB & IIB & IIB & 8 & & $\mathrm{R}(\mathrm{RR})$ \\
\hline 16 & None & & IA & $\mathrm{IA}$ & IA & IA & IA & IA & IA & IIB & 8 & re-SAH 2007 & $\mathrm{CH}$ \\
\hline 17 & $\mathrm{H}$ & & IA & IIA & IIA & IIA & IIIA & IIIA & IIIA & & 7 & VNS-impl 2003, expl 2012 & I \\
\hline 18 & None & & $\mathrm{IA}$ & $\mathrm{IA}$ & IIA & IIA & $\mathrm{IA}$ & IA & IA & & 7 & & $\mathrm{CH}$ \\
\hline 19 & $\mathrm{MS} / \mathrm{P}$ & & IB & IB & IB & IA & IA & IA & IA & & 7 & re-SAH 2010 & $\mathrm{D}$ \\
\hline 20 & None & & $\mathrm{IB}$ & IB & IB & IB & IB & IB & IB & & 7 & & $U$ \\
\hline 21 & $\mathrm{MS} / \mathrm{N}$ & PD & IA & ID & ID & ID & ID & IA & & & 6 & & $\mathrm{R}$ \\
\hline 22 & None & & $\mathrm{IA}$ & IIA & ATL & & & & & & 2 & ATL-QA & N/A R \\
\hline 23 & None & & IA & IA & IA & IIA & IC & IC & & & 6 & & $\mathrm{CH}$ \\
\hline 24 & None & & IB & IB & $\mathrm{IA}$ & IB & IB & IB & & & 6 & & $U$ \\
\hline 25 & None & & IA & IA & IA & IA & IA & ID & & & 6 & & $\mathrm{R}$ \\
\hline 26 & $\mathrm{ICH}$ & & $\mathrm{IA}$ & IA & $\mathrm{IA}$ & $\mathrm{IA}$ & $\mathrm{IA}$ & $\mathrm{IA}$ & & & 6 & & $\mathrm{D}$ \\
\hline 27 & None & & $\| \mathrm{A}$ & $\| \mathrm{A}$ & IC & IC & ID & IC & & & 6 & & $U$ \\
\hline 28 & None & & IA & IB & IB & IB & IA & IA & & & 6 & & $U$ \\
\hline 29 & None & & IB & IA & IB & IB & IB & IB & & & 6 & Cavernoma-LGK & $U$ \\
\hline 30 & $M S / N$ & & IIB & IIB & IA & IA & IA & IA & & & 6 & & $U$ \\
\hline 31 & $\mathrm{ICH}, \mathrm{MS} / \mathrm{N}$ & & IB & IB & IB & IB & IB & IB & & & 6 & & $U$ \\
\hline 32 & None & & IA & $\mathrm{IA}$ & IA & IA & IA & & & & 5 & & $U$ \\
\hline 33 & $\mathrm{H}$ & & IVB & ATL & & & & & & & 1 & & $N / A(R, R R)$ \\
\hline 34 & None & & IA & IA & IB & IB & IA & & & & 5 & & U \\
\hline 35 & None & & IIIA & died & & & & & & & I & & N/A (U) \\
\hline 36 & None & & $\mathrm{IA}$ & IA & IA & IA & IA & & & & 5 & & $\mathrm{R}$ \\
\hline 37 & $\mathrm{OH}, \mathrm{ICH}$ & & IB & IB & IB & IB & IB & & & & 5 & & $U$ \\
\hline 38 & None & PD & IIA & IIA & IIA & IIIB & IIIB & & & & 5 & & $U$ \\
\hline 39 & None & & IA & IA & $\mathrm{IA}$ & IA & IA & & & & 5 & & $\mathrm{R}$ \\
\hline 40 & $\mathrm{H}$ & & IA & IA & ID & $\mathrm{IA}$ & $\mathrm{IA}$ & & & & 5 & & $\mathrm{CH}(\mathrm{RR})$ \\
\hline 41 & None & & IVA & IVB & IVB & IVB & IVB & & & & 5 & & $\mathrm{~N} / \mathrm{A}, \mathrm{U}$ \\
\hline
\end{tabular}

(Continued) 
Table S2 (Continued)

\begin{tabular}{|c|c|c|c|c|c|c|c|c|c|c|c|c|c|}
\hline$\overline{P t}$ no & Early c & Late c & FU-I & FU-2 & FU-3 & FU-4 & FU-5 & FU-6 & FU-7 & FU-8 & Follow-up & Notes & Medication \\
\hline$\overline{42}$ & None & & IA & IA & IA & IA & IIIB & & & & 5 & & U (RR) \\
\hline 43 & None & & IIIB & IIIB & IIIB & IIIB & & & & & 4 & & I \\
\hline 44 & $\mathrm{H}$ & & IIIA & IIIA & ATL & & & & & & 2 & Cavernoma? & $\mathrm{N} / \mathrm{A}(\mathrm{U})$ \\
\hline 45 & None & & $\| \mathrm{A}$ & IB & $\mathrm{IA}$ & IA & & & & & 4 & & $U$ \\
\hline 46 & None & & IVB & IVB & IVB & IVB & & & & & 4 & & $\mathrm{CH}$ \\
\hline 47 & None & & IA & IB & IB & IB & & & & & 4 & & $U$ \\
\hline 48 & None & & IIB & IIB & IIB & IIB & & & & & 4 & & $U$ \\
\hline 49 & None & & IB & IB & IB & IB & & & & & 4 & ATL 2005 & $U$ \\
\hline 50 & None & & IA & $\mathrm{IA}$ & $\mathrm{IA}$ & IA & & & & & 4 & & $U$ \\
\hline 51 & None & & IB & IB & IB & IB & & & & & 4 & & $U$ \\
\hline 52 & None & & IIIA & IIIA & IIIA & IIIA & & & & & 4 & & 1 \\
\hline 53 & None & & IA & $\mathrm{IA}$ & IA & & & & & & 3 & & $U$ \\
\hline 54 & None & & IA & $\mathrm{IA}$ & $\mathrm{IA}$ & & & & & & 3 & & $\mathrm{R}$ \\
\hline 55 & None & & IIB & IIB & IIB & & & & & & 3 & & $\mathrm{R}$ \\
\hline 56 & None & IIP & IIA & IIIA & IIIA & & & & & & 3 & & $\mathrm{CH}$ \\
\hline 57 & None & & IB & IB & IB & & & & & & 3 & & $U$ \\
\hline 58 & None & PD & IB & IB & IB & & & & & & 3 & & $U$ \\
\hline 59 & None & & IB & IB & IB & & & & & & 3 & & $U$ \\
\hline 60 & None & & IA & IA & IB & & & & & & 3 & & $U$ \\
\hline 61 & None & & IIA & IIIA & died & & & & & & 2 & & $\mathrm{~N} / \mathrm{A}(\mathrm{U})$ \\
\hline
\end{tabular}

Note: Data printed in red show Engel scores at last follow-up and reasons for their presentation earlier than expected.

Abbreviations: ATL-QA, quadrantanopia after ATL; BP, broken tip of the probe; CC, cortical contussion; CH, changed; D, depression; DP, transient dysphasia; Early c, early complications; FU, follow-up; H, headache; I, increased; ICH, intracerebral hematoma; IIP, interictal psychosis; Late c, late complications; LGK, treatment with Leksell gamma knife; M, meningitis; MS/N, meningeal syndrome, lumbar tap not performed; MS/P MS/P, meningeal syndrome, lumbar tap performed; N/A, not available; OH, obstructive hydrocephalus; PA, pituitary adenoma; PD, decompensated personality disorder; PE, pulmonary embolism; PNES, psychogenic nonepileptic seizures; Pt no, patient number; $\mathrm{QA}$, quadrantanopia; $\mathrm{R}$, reduced; RR, relapse; SAH, re-thermolesion; SDH, subdural haematoma; $\mathrm{U}$, unchanged; VNS, vagus nerve stimulation.

\section{Publish your work in this journal}

Neuropsychiatric Disease and Treatment is an international, peerreviewed journal of clinical therapeutics and pharmacology focusing on concise rapid reporting of clinical or pre-clinical studies on a range of neuropsychiatric and neurological disorders. This journal is indexed on PubMed Central, the 'PsycINFO' database and CAS, and is the official journal of The International Neuropsychiatric Association (INA). The manuscript management system is completely online and includes a very quick and fair peer-review system, which is all easy to use. Visit http://www.dovepress.com/testimonials.php to read real quotes from published authors. 